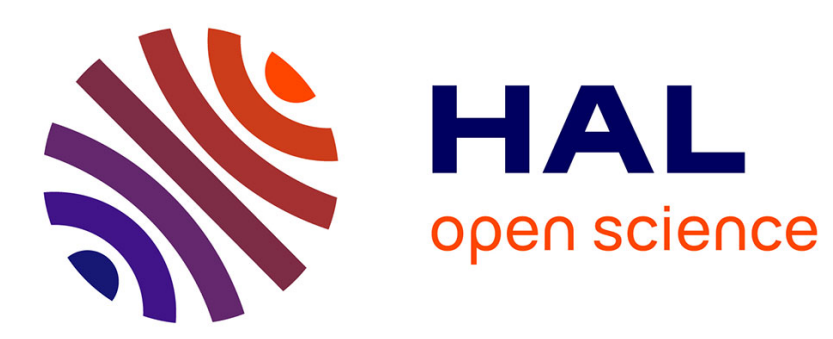

\title{
Computing a Guaranteed Approximation of the Zone Explored by a Robot
}

\author{
Benoît Desrochers, Luc Jaulin
}

\section{To cite this version:}

Benoît Desrochers, Luc Jaulin. Computing a Guaranteed Approximation of the Zone Explored by a Robot. IEEE Transactions on Automatic Control, 2017, 62 (1), pp.425 - 430. 10.1109/TAC.2016.2530719 . hal-01298366

\section{HAL Id: hal-01298366 \\ https://hal.science/hal-01298366}

Submitted on 1 Feb 2018

HAL is a multi-disciplinary open access archive for the deposit and dissemination of scientific research documents, whether they are published or not. The documents may come from teaching and research institutions in France or abroad, or from public or private research centers.
L'archive ouverte pluridisciplinaire HAL, est destinée au dépôt et à la diffusion de documents scientifiques de niveau recherche, publiés ou non, émanant des établissements d'enseignement et de recherche français ou étrangers, des laboratoires publics ou privés. 


\title{
Computing a Guaranteed Approximation of the Zone Explored by a Robot
}

\author{
Benoît Desrochers ${ }^{1,2}$ and Luc Jaulin ${ }^{2}$
}

\begin{abstract}
This paper deals with the guaranteed characterization of the part of the space that has been explored by a robot. The main difficulty of the problem is to take into account the uncertainty associated with the trajectory and the fact that the dimension of the visible space at time $t$ may be smaller than that of the workspace. An example involving an experiment made with an actual underwater robot is presented in order to illustrate the efficiency of the approach.
\end{abstract}

\section{INTRODUCTION}

In this paper, we consider a robot moving inside an unknown environment in a dead reckoning manner, i.e., using the proprioceptive sensors only. As it is the case for most industrial underwater robots, the robot is equipped with some exteroceptive sensors (such as sonars or cameras) that are used for exploration only and not for navigation. After the mission, it has to find which part of the environment it has explored, taking into account the uncertainty on the localization. We assume that the interpretation of exploration sensors is not reliable enough to allow us using SLAM techniques such as in [11] or [5]. When the mission is short and the quality of the proprioceptive sensors is good, occupancy map techniques [4] can be used to mark all points that have been observed and a probability of being explored can be associated with each part of the space [15]. Now, in practice, due to the state noise, the prediction of the location of the robot is getting less accurate and we have to take into account these uncertainties properly. We are in a typical situation where the uncertainty is combined with inaccuracy [3]. The unknown variable of our problem is the explored set $\mathbb{Z}$, and characterizing uncertain sets with classical probabilistic methods requires elaborated mathematical tools such as random sets [12]. These random sets have already been used in the context of robot mapping (see [13]) but the approach is limited to finite sets. In a set membership approach, an uncertain set $\mathbb{Z}$ can be bracketed by two sets $\mathbb{Z}^{-}$and $\mathbb{Z}^{+}$such that $\mathbb{Z}^{-} \subset \mathbb{Z} \subset \mathbb{Z}^{+}$. This representation is particularly adapted to represent uncertain maps [10]. Interval-type uncertainties can easily be propagated through nonlinear functions (see e.g., [6]) or nonlinear state equations, as shown in [1] to get an inner and an outer characterization of the reachable space or in [8] for SLAM involving unstructured maps.

\footnotetext{
${ }^{1}$ Direction Générale de l'Armement, Techniques Navales Brest, BCRM de Brest, 29240 Brest Cedex 9.

${ }^{2}$ ENSTA-Bretagne, LabSTICC, IHSEV, OSM, 2 rue François Verny, 29806 Brest.

benoit.desrocherseensta-bretagne.org,

lucjaulin@gmail.com
}

The paper is organized as follows. Section II defines the notion of explored zone and proposes a formalization of the problem. Section III extends the formalism in order to take into account the uncertainty associated with the trajectory of the robot. Section IV] gives the new algorithm which encloses the explored zone $\mathbb{Z}$ between two subpavings (union of boxes). An experiment involving the underwater robot Daurade is treated in Section $\mathrm{V}$ in order to validate the feasibility of the approach. A conclusion is then given on Section VI.

\section{Problem Statement}

The problem to be considered here is the characterization of the explored zone $\mathbb{Z}$ of a robot. The set $\mathbb{Z}$ is defined by

$$
\left\{\begin{array}{cc}
\text { (i) } & \dot{\mathbf{x}}=\mathbf{f}(\mathbf{x}, \mathbf{u}), \mathbf{x}(0)=\mathbf{x}_{0}, \mathbf{u}(t) \in[\mathbf{u}](t) \\
\text { (ii) } & \mathbb{Z}=\bigcup_{t \geq 0} \mathbb{V}(\mathbf{x}(t))
\end{array}\right.
$$

where (i) describes the evolution of the robot and (ii) defines the explored zone $\mathbb{Z}$. In the state equation (i) of the robot, $\mathbf{x} \in \mathbb{R}^{n}$ is the state vector and $\mathbf{u}$ is the input vector. In order to take into account some state noise, we assume that, for all $t$, a box $[\mathbf{u}](t)$ which contains $\mathbf{u}(t)$ is available. Moreover, the initial condition $\mathbf{x}_{0}$ is assumed to be known. For each $t$, a scanner on the robot is able to observe a part of its environment. More precisely, for each $t$, there exists a subset of the environment $\mathbb{V}(\mathbf{x}(t)) \subset \mathbb{R}^{q}, q \in\{2,3\}$ that is visible by the robot. This set is called the visible set (see, e.g., [7] in the context of localization). Note that our robot does not use any exteroceptive sensors for state estimation, for localization or to control its trajectory. It is only able to estimate its position from the proprioceptive sensors $\mathbf{u}$ with a given bounded accuracy. Equivalently, we consider that the scan sensors of the robot are used to collect data for exploration that will only be analyzed after the mission by some human users. The following examples illustrate how the visible sets could be defined in practice.

Example 1. Consider a robot, the pose of which is $\mathbf{x}=$ $(x, y, \theta)$, moving in a plane. This robot is able to scan the environment up to a distance of 3 meters in front of it inside a cone of $\pm \frac{\pi}{6} \mathrm{rad}$. Therefore, the visible set $\mathbb{V}(\mathbf{x})$ contains all $\mathbf{z} \in \mathbb{R}^{2}$ which satisfy the following inequalities

$$
\begin{cases}\left(z_{1}-x\right)^{2}+\left(z_{2}-y\right)^{2} & \leq 9 \\ \cos \theta \cdot\left(z_{1}-x\right)+\sin \theta \cdot\left(z_{2}-y\right) & \geq \cos \frac{\pi}{6} .\end{cases}
$$

Example 2. We consider the robot of Example 1 except that now, this robot is able to scan only points that are exactly 
on its right at a distance in $[2,3]$. Then $\mathbb{V}(\mathbf{x})$ is the set of all $\mathbf{z} \in \mathbb{R}^{2}$ such that

$$
\begin{cases}\left(z_{1}-x\right)^{2}+\left(z_{2}-y\right)^{2} & \in[4,9] \\ \cos \theta \cdot\left(z_{1}-x\right)+\sin \theta \cdot\left(z_{2}-y\right) & =0 \\ \cos \theta \cdot\left(z_{2}-y\right)-\sin \theta\left(z_{1}-x\right) & \leq 0\end{cases}
$$

Example 3. The same robot is now able to scan only points that are exactly on its right at a distance of 3 meters. Then $\mathbb{V}(\mathbf{x})$ is the set of all $\mathbf{z} \in \mathbb{R}^{2}$ such that

$$
\begin{cases}\left(z_{1}-x\right)^{2}+\left(z_{2}-y\right)^{2}-3^{2} & =0 \\ \cos \theta \cdot\left(z_{1}-x\right)+\sin \theta \cdot\left(z_{2}-y\right) & =0 \\ \cos \theta \cdot\left(z_{2}-y\right)-\sin \theta\left(z_{1}-x\right) & \leq 0\end{cases}
$$

As motivated by these three examples, we may consider three different types of exploration.

1) Patch exploration [2]. The visible sets $\mathbb{V}(\mathbf{x})$ have a dimension $q$, as for Example 1. In this case, $\operatorname{dim}(\mathbb{Z})=q$ and it will be possible to compute an inner and an outer approximation of $\mathbb{Z}$. This type of exploration exists when the sensor is a camera: a 3D zone is explored for each $t$.

2) Sweep exploration. The visible sets $\mathbb{V}(\mathbf{x})$ have a dimension $q-1$, as for Example 2. Thus, $\mathbb{Z}$ has a dimension of $q$. Again, it will be possible [14] to bracket $\mathbb{Z}$ from both inside and outside. This is the case when the robot is equipped with a side scan sonar, a multi-beam echo sounder or an airborne LiDAR.

3) Pen exploration. The dimension of $\mathbb{V}(\mathbf{x})$ is smaller than $q-2$, as for Example 3, and thus $\operatorname{dim}(\mathbb{Z}) \leq q-1$. It will neither be possible to get an inner approximation for $\mathbb{Z}$ nor to prove that a given point is actually inside $\mathbb{Z}$. This is the case when the robot is equipped with a laser rangefinder and takes one range measurement every second.

When the whole trajectory $\mathbf{x}(t)$ is known, the set $\mathbb{Z}$ is clearly defined. Different techniques could be thought in order to characterize the set $\mathbb{Z}$. For instance, we could use subpavingbased methods [9] or occupancy grid approaches. Now, in this paper, the trajectory of the robot is not known precisely and the set $\mathbb{Z}$ becomes uncertain which makes the problem much more difficult [15].

\section{UNCERTAIN EXPLORED ZONES}

When the trajectory $\mathbf{x}(\cdot)$ is uncertain, the explored zone $\mathbb{Z}$ cannot be approximated with an arbitrary accuracy. In a probabilistic context, we can associate to each $\mathbf{z} \in \mathbb{R}^{q}$ a probability of being explored. Now, even if this probability can be estimated using Monté-Carlo methods, due to the large (here infinite) dimension of the set of trajectories to be explored, the computational burden is high which makes probabilistic methods not so attractive. Denote by $\mathcal{X}(\cdot)$, the set of all feasible trajectories, i.e., the trajectories $\mathbf{x}(\cdot)$ consistent with (1) and with the initial condition $\mathbf{x}(0)$. It is important not to make the confusion between $\mathbf{x}(\cdot)$ which is a trajectory and $\mathbf{x}(t)$ which is a vector of $\mathbb{R}^{n}$. We define the two following sets

$$
\begin{aligned}
& \mathbb{Z}^{-}=\bigcap_{\mathbf{x}(\cdot) \in \mathcal{X}(\cdot)} \bigcup_{t \geq 0} \mathbb{V}(\mathbf{x}(t)) \\
& \mathbb{Z}^{+}=\bigcup_{\mathbf{x}(\cdot) \in \mathcal{X}(\cdot)} \bigcup_{t \geq 0} \mathbb{V}(\mathbf{x}(t))
\end{aligned}
$$
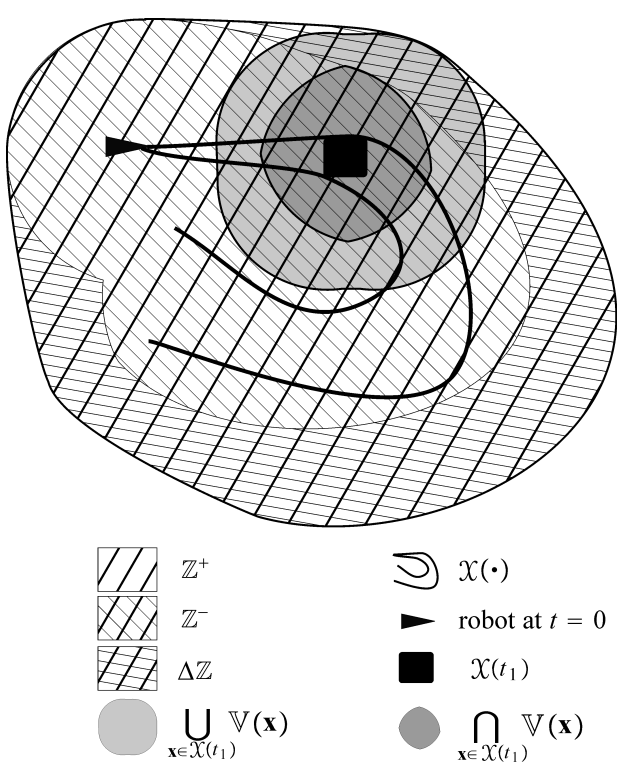

Fig. 1. The space is partitioned into three zones: $\overline{\mathbb{Z}^{+}}$is the dark zone, $\overline{\mathbb{Z}^{-}} \cap$ $\mathbb{Z}^{+}$is the penumbra and $\mathbb{Z}^{-}$is the clear zone

The set $\mathbb{Z}^{-}$is called the certainly explored zone or the clear zone. It corresponds to the set of all points $\mathrm{z}$ of the environment that have certainly been seen by the robot taking into account that its trajectory $\mathbf{x}(\cdot)$ is feasible. The complementary set $\overline{\mathbb{Z}^{+}}$of $\mathbb{Z}^{+}$is called the certainly unexplored zone or the dark zone. The set $\mathbb{Z}^{+}$is called non-dark zone. Moreover, we define the penumbra as the set $\Delta \mathbb{Z}=\mathbb{Z}^{+} \backslash \mathbb{Z}^{-}$(see Figure 11. It corresponds to the set of all $\mathbf{z}$ that have been seen by some feasible trajectories and not seen by some other feasible trajectories. Figure 1 illustrates a situation of a patch exploration where the patch is a disk. At time $t_{1}$ the robot knows that it is inside the black box. Taking this uncertainty into account, it concludes that the dark gray zone is certainly illuminated (i.e., all corresponding points $\mathbf{z}$ are certainly seen at time $t_{1}$ ) and that all points inside the light gray zone have possibly been seen at time $t_{1}$. The unknown explored zone $\mathbb{Z}$ obviously satisfies

$$
\mathbb{Z}^{-} \subset \mathbb{Z} \subset \mathbb{Z}^{+}
$$

Note that the clear zone $\mathbb{Z}^{-}$is larger than the union of the certainly visible sets, i.e.,

$$
\underbrace{\bigcup_{t \geq 0} \bigcap_{\mathbf{x} \in \mathcal{X}(t)} \mathbb{V}(\mathbf{x})}_{\{\mathbf{z} \mid \exists t \forall \mathbf{x} \in \mathcal{X}(t), \mathbf{z} \in \mathbb{V}(\mathbf{x})\}} \subset \mathbb{Z}^{-}=\underbrace{\bigcap_{\mathbf{x}(\cdot) \in \mathcal{X}(\cdot)} \bigcup_{t \geq 0} \mathbb{V}(\mathbf{x}(t))}_{\{\mathbf{z} \mid \forall \mathbf{x}(\cdot) \in \mathcal{X}(\cdot), \exists t, \mathbf{z} \in \mathbb{V}(\mathbf{x})\}} .
$$

This inclusion is due to the fact that, $\cap$ and $\cup$ do not commute in general. The consequence of this non commutativity is illustrated by Figure 2 where two different trajectories for the robot are represented. The pies correspond to the set of all $\mathbf{z}$ that have been seen by the robot at times $t_{1}$ and $t_{2}$ for the two trajectories. To characterize $\mathbb{Z}^{-}$, it is not sufficient to compute the union of all certainly visible sets; we also need to add all cross intersections. For instance, whatever is the true trajectory among $\mathbf{x}^{a}$ or $\mathbf{x}^{b}$, we are certain that all points of the dark gray set (at the intersection between the two opposite pies) in the 


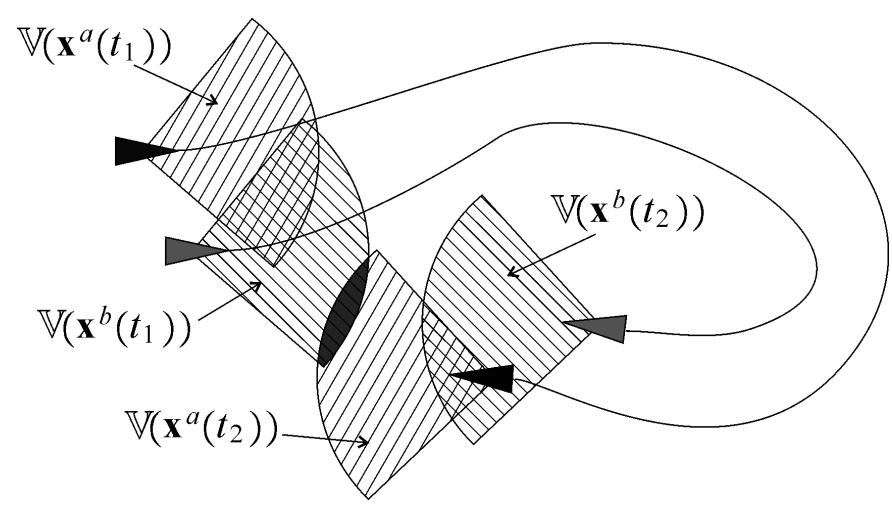

Fig. 2. Even if we do not know which one of the two trajectories is the true one, we are certain that the gray set has been explored; the pies correspond to illuminated zones.

figure have been explored. These cross intersections are not taken into account by existing methods [2].

Now, for sweep exploration, the dimension of the visible sets is $q-1$ and the certainly visible sets $\bigcap_{\mathbf{x} \in \mathcal{X}(t)} \mathbb{V}(\mathbf{x})$ become empty. All the information about $\mathbb{Z}^{-}$can thus only be obtained from these cross intersections, i.e., by taking into account different $t$ and different trajectories all together or equivalently, by considering the right hand side of (7) for the characterization of $\mathbb{Z}^{-}$.

\section{CHARACTERIZATION OF THE EXPLORED ZONE}

We now focus our attention on the sweep exploration where visible sets have the form

$$
\mathbb{V}(\mathbf{x})=\left\{\mathbf{z} \in \mathbb{R}^{q} \mid \varphi(\mathbf{z}, \mathbf{x})=0 \text { and } \psi(\mathbf{z}, \mathbf{x}) \leq 0\right\} .
$$

In this formula, $\varphi: \mathbb{R}^{p} \times \mathbb{R}^{n} \rightarrow \mathbb{R}$ is the visibility function which is assumed to be continuous and $\psi: \mathbb{R}^{p} \times \mathbb{R}^{n} \rightarrow \mathbb{R}$ models the scope of the sensor. Examples 1, 2, 3 of Section III provide an illustration of these functions.

This section proposes a new algorithm able to bracket the explored zone between two subpavings (union of nonoverlapping boxes). More precisely, the workspace will be partitioned into 4 types of boxes: (a) the clear boxes that have been proved to be inside $\mathbb{Z}^{-}$, (b) the dark boxes that have been proved to be outside $\mathbb{Z}^{+}$, (c) the penumbra boxes which are neither dark nor clear and (d) the boxes which have not be classified yet. When a box cannot be classified, it is bisected into two non overlapping subboxes. To ensure the convergence of the algorithm, boxes which have a width smaller than a small given value are not bisected.

To classify boxes (clear, dark, penumbra), we need first to get a guaranteed enclosure of the set of feasible trajectories $\mathcal{X}(\cdot)$. This can be done using interval guaranteed integration [18], [17], [16]. These techniques make it possible to compute a tube (i.e., a function $[\mathbf{x}](\cdot)$ mapping $\mathbb{R}$ into $\mathbb{R}^{n}$, the set of boxes of $\mathbb{R}^{n}$ ) which encloses the set of trajectories $\mathcal{X}(\cdot)$. In what follows, we shall thus assume that the trajectory $\mathbf{x}(\cdot)$ is inside a tube denoted by $[\mathbf{x}](\cdot)$. As a consequence, from (5), we have

$$
\bigcap_{\mathbf{x}(\cdot) \in[\mathbf{x}](\cdot)} \bigcup_{t \geq 0} \mathbb{V}(\mathbf{x}(t)) \subset \mathbb{Z} \subset \bigcup_{\mathbf{x}(\cdot) \in[\mathbf{x}](\cdot)} \bigcup_{t \geq 0} \mathbb{V}(\mathbf{x}(t)) .
$$

\section{A. Notion of Logic}

The presentation of the tests that will be used to classify the boxes of the workspace requires some notions on logic, involving quantifiers. Some useful rules are now presented. If $A$ is a predicate, if $\varphi(\cdot)$ is continuous, if $\mathbf{x}(\cdot)$ is a trajectory and $[\mathbf{x}](\cdot)$ is a tube, we have

$$
\begin{gathered}
\exists \mathbf{x}(\cdot) \in[\mathbf{x}](\cdot), \forall t, A(\mathbf{x}(t)) \\
\Leftrightarrow \forall t, \exists \mathbf{a} \in[\mathbf{x}](t), A(\mathbf{a}) \\
\exists t, \varphi(t)=0 \wedge \psi(t) \leq 0 \\
\Leftrightarrow \exists t_{1} \exists t_{2},\left\{\begin{array}{l}
\varphi\left(t_{1}\right) \varphi\left(t_{2}\right) \leq 0 \\
\psi\left(\left[t_{1}, t_{2}\right]\right) \subset \mathbb{R}^{-}
\end{array}\right. \\
\forall \mathbf{x}(\cdot) \in[\mathbf{x}](\cdot), \exists t, A(\mathbf{x}(t)) \\
\Leftrightarrow \exists t, \forall \mathbf{a} \in[\mathbf{x}](t), A(\mathbf{a}) .
\end{gathered}
$$

\section{B. Clarity Test}

The clarity test aims at proving that a box $[\mathbf{z}]$ has been explored whatever the uncertainties are. This test is based on the following theorem.

Theorem 1. Given an interval $\left[t_{1}, t_{2}\right] \subset \mathbb{R}^{+}$and a tube $[\mathbf{x}](\cdot)$, we have:

$$
\left.\begin{array}{c}
\varphi\left(\mathbf{z},[\mathbf{x}]\left(t_{1}\right)\right) \cdot \varphi\left(\mathbf{z},[\mathbf{x}]\left(t_{2}\right)\right) \subset \mathbb{R}^{-} \\
\wedge \psi\left(\mathbf{z},[\mathbf{x}]\left(\left[t_{1}, t_{2}\right]\right)\right) \subset \mathbb{R}^{-}
\end{array}\right\} \Rightarrow \mathbf{z} \in \mathbb{Z}^{-}
$$

Proof: Consider a point $\mathbf{z} \in \mathbb{R}^{q}$. The left hand side of is equivalent to

$$
\begin{aligned}
& \forall \mathbf{x}(\cdot) \in[\mathbf{x}](\cdot), \quad\left(\varphi\left(\mathbf{z}, \mathbf{x}\left(t_{1}\right)\right) \cdot \varphi\left(\mathbf{z}, \mathbf{x}\left(t_{2}\right)\right) \leq 0\right) \\
& \wedge \psi\left(\mathbf{z}, \mathbf{x}\left(\left[t_{1}, t_{2}\right]\right)\right) \subset \mathbb{R}^{-}
\end{aligned}
$$

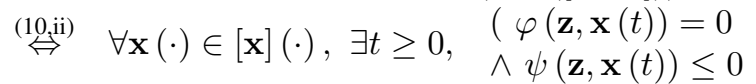

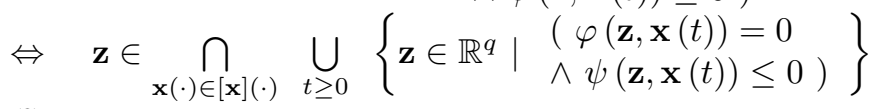

$$
\begin{aligned}
& \stackrel{8}{g} \mathbf{z} \in \bigcap_{\mathbf{x}(\cdot) \in[\mathbf{x}](\cdot)} \bigcup_{t \geq 0} \mathbb{V}(\mathbf{x}(t)) \\
& \text { 乌 } \mathbf{z} \in \mathbb{Z}^{-} \text {. }
\end{aligned}
$$

Clarity test. Given $t_{1}, t_{2} \geq 0$ and inclusion functions $[\varphi],[\psi]$ for $\varphi, \psi$, we define the clarity test as:

$T_{\text {clarity }}([\mathbf{z}],[\mathbf{x}](\cdot)):\left\{\begin{array}{c}{[\varphi]\left([\mathbf{z}],[\mathbf{x}]\left(t_{1}\right)\right) \cdot[\varphi]\left([\mathbf{z}],[\mathbf{x}]\left(t_{2}\right)\right) \subset \mathbb{R}^{-}} \\ \wedge[\psi]\left([\mathbf{z}],[\mathbf{x}]\left(\left[t_{1}, t_{2}\right]\right)\right) \subset \mathbb{R}^{-}\end{array}\right.$

From Theorem 1] we have

$$
T_{\text {clarity }}([\mathbf{z}],[\mathbf{x}](\cdot)) \Rightarrow[\mathbf{z}] \subset \mathbb{Z}^{-} .
$$

An illustration of this proposition is given by Figure 3 . We choose the pairs $\left(t_{1}, t_{2}\right)$ as in the figure, i.e., such that $t_{2}-t_{1}$ are small and such that

$$
[\varphi]\left([\mathbf{z}],[\mathbf{x}]\left(t_{1}\right)\right) \cdot[\varphi]\left([\mathbf{z}],[\mathbf{x}]\left(t_{2}\right)\right) \subset \mathbb{R}^{-} .
$$

Note that the test also concludes for the pair $\left(t_{5}, t_{6}\right)$ but fails for $\left(t_{3}, t_{4}\right)$. Since here, the test concludes for at least one pair, we get that the whole box $[\mathbf{z}]$ has been explored. 


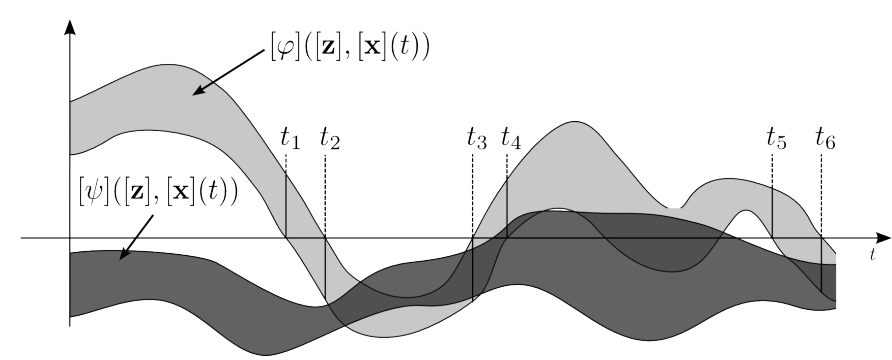

Fig. 3. The fact that $\varphi$ changes its sign inside $\left[t_{1}, t_{2}\right]$ whereas $\psi$ remains negative implies that $[\mathbf{z}] \subset \mathbb{Z}$

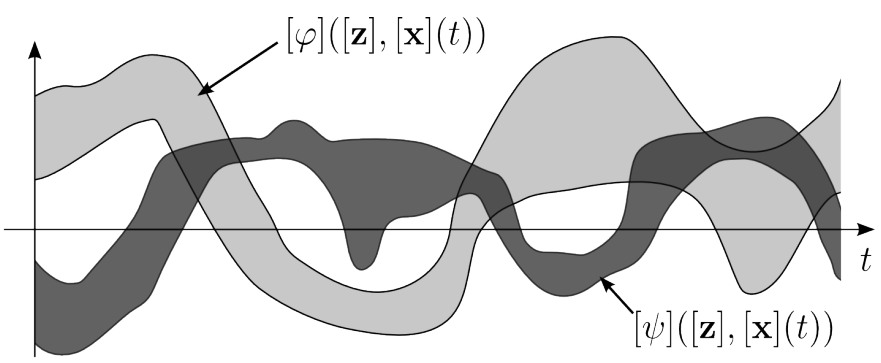

Fig. 4. This configuration for the tubes $[\varphi](\cdot)$ and $[\psi](\cdot)$ allows us to conclude that $[\mathbf{z}]$ is dark

\section{Dark Test}

The dark test aims at proving that no point in the box $[\mathbf{z}]$ has been explored whatever the uncertainties are. The dark test is based on the following theorem.

Theorem 2. If $[\mathbf{x}](\cdot)$ is a tube, then we have

$$
\begin{gathered}
\forall t \geq 0, \forall \mathbf{x} \in[\mathbf{x}](t),(\varphi(\mathbf{z}, \mathbf{x}) \neq 0) \\
\vee(\psi(\mathbf{z}, \mathbf{x})>0) \Rightarrow \mathbf{z} \notin \mathbb{Z}^{+} .
\end{gathered}
$$

Proof. The proof is by contradiction, i.e., we show that assuming $\mathbf{z} \in \mathbb{Z}^{+}$implies that the left hand side of $(14)$ is false. We have

$$
\begin{aligned}
& \mathbf{z} \in \mathbb{Z}^{+} \\
& \stackrel{\text { 9. }}{\Rightarrow} \mathbf{z} \in \bigcup_{\mathbf{x}(\cdot) \in[\mathbf{x}](\cdot)} \bigcup_{t \geq 0} \mathbb{V}(\mathbf{x}(t))
\end{aligned}
$$

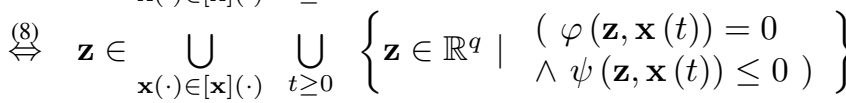

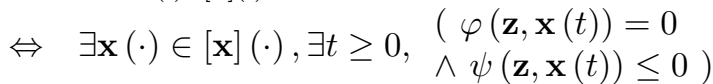

which corresponds to the negation of left hand side of $(14)$.

Dark test. Given two inclusion functions $[\varphi],[\psi]$ for $\varphi, \psi$. Define the dark test as

$$
T_{\text {dark }}([\mathbf{z}],[\mathbf{x}](\cdot)): \forall t \geq 0,\left\{\begin{array}{c}
(0 \notin[\varphi]([\mathbf{z}],[\mathbf{x}](t))) \\
\vee\left([\psi]([\mathbf{z}],[\mathbf{x}](t)) \subset \mathbb{R}^{+}\right) .
\end{array}\right.
$$

From Theorem 2, we have

$$
T_{\text {dark }}([\mathbf{z}],[\mathbf{x}](\cdot)) \Rightarrow[\mathbf{z}] \cap \mathbb{Z}^{+} .
$$

An illustration of this proposition is given by Figure 4 In the situation represented in this figure, $\forall t \geq 0, \forall \mathbf{x} \in[\mathbf{x}](t), \forall \mathbf{z} \in$ $[\mathbf{z}]$, either $\varphi(\mathbf{z}, \mathbf{x}) \neq 0$ or $(\psi(\mathbf{z}, \mathbf{x})>0)$, which means that no point inside $\mathbf{z} \in[\mathbf{z}]$ could have been seen.

\section{Penumbra Test}

Proving that a point $\mathbf{z}$ belongs to the penumbra $\Delta \mathbb{Z}$ is difficult and not so useful. The main interest to have an inclusion test for the penumbra is to limit the computation burden. Indeed, if $[\mathbf{z}] \subset \Delta \mathbb{Z}$ then we will never be able to prove that $[\mathbf{z}] \subset \mathbb{Z}^{-}$or to prove that $[\mathbf{z}] \cap \mathbb{Z}^{+}=\emptyset$, and thus there is no need to bisect. Now, instead of proving that $[\mathbf{z}] \subset \Delta \mathbb{Z}$, we propose here to test either the darkness test or the clarity test always fail for all subbox of $[\mathbf{z}]$. In such a case, $[\mathbf{z}]$ will not be bisected. In such a situation, we shall say that $[\mathbf{z}]$ satisfies the penumbra test.

Theorem 3. Given $\mathbf{x}(\cdot) \in[\mathbf{x}](\cdot)$ and $\mathbf{z} \in[\mathbf{z}]$. If

$$
\forall t,\left\{\begin{array}{l}
\varphi(\mathbf{z}, \mathbf{x}(t)) \neq 0 \\
\vee \psi(\mathbf{z}, \mathbf{x}(t))>0
\end{array} \Rightarrow \neg T_{\text {clarity }}([\mathbf{x}](.),[\mathbf{z}]) .\right.
$$

Proof. The proof is by contradiction. Assume that $T_{\text {clarity }}([\mathbf{x}](),.[\mathbf{z}])$ is true, i.e.,

$$
\left\{\begin{array}{c}
{[\varphi]\left([\mathbf{z}],[\mathbf{x}]\left(t_{1}\right)\right) \cdot[\varphi]\left([\mathbf{z}],[\mathbf{x}]\left(t_{2}\right)\right) \subset \mathbb{R}^{-}} \\
\wedge[\psi]\left([\mathbf{z}],[\mathbf{x}]\left(\left[t_{1}, t_{2}\right]\right)\right) \subset \mathbb{R}^{-} .
\end{array}\right.
$$

Since $\mathbf{x}(\cdot) \in[\mathbf{x}](\cdot), \mathbf{z} \in[\mathbf{z}]$ and since $[\psi],[\varphi]$ are inclusion functions of $\psi, \varphi$, we get

$$
\left\{\begin{array}{c}
\varphi\left(\mathbf{z}, \mathbf{x}\left(t_{1}\right)\right) \cdot \varphi\left(\mathbf{z}, \mathbf{x}\left(t_{2}\right)\right) \in \mathbb{R}^{-} \\
\wedge \psi\left(\mathbf{z}, \mathbf{x}\left(\left[t_{1}, t_{2}\right]\right)\right) \subset \mathbb{R}^{-} .
\end{array}\right.
$$

From 10 ii), we get that there exists $t_{0} \in\left[t_{1}, t_{2}\right]$ such that

$$
\varphi\left(\mathbf{z}, \mathbf{x}\left(t_{0}\right)\right)=0 \wedge \psi\left(\mathbf{z}, \mathbf{x}\left(t_{0}\right)\right) \leq 0
$$

which is inconsistent with the left hand side of (17).

Theorem 4. Given $\mathbf{x}(\cdot) \in[\mathbf{x}](\cdot), \mathbf{z} \in[\mathbf{z}]$ and $t_{1}, t_{2} \geq 0$. We have:

$$
\left.\begin{array}{c}
\varphi\left(\mathbf{z}, \mathbf{x}\left(t_{1}\right)\right) \cdot \varphi\left(\mathbf{z}, \mathbf{x}\left(t_{2}\right)\right) \leq 0 \\
\wedge \forall t \in\left[t_{1}, t_{2}\right], \psi(\mathbf{z}, \mathbf{x}(t)) \leq 0
\end{array}\right\} \Rightarrow \neg T_{\text {dark }}([\mathbf{x}](\cdot),[\mathbf{z}]) .
$$

Proof. The proof is by contradiction. Assume that $T_{\text {dark }}([\mathbf{x}](\cdot),[\mathbf{z}])$ is true. From $[15$, this amounts to saying that

$$
\forall t \geq 0,\left\{\begin{array}{c}
(0 \notin[\varphi]([\mathbf{z}],[\mathbf{x}](t))) \\
\vee\left([\psi]([\mathbf{z}],[\mathbf{x}](t)) \subset \mathbb{R}^{+}\right) .
\end{array}\right.
$$

Since $\mathbf{x}(\cdot) \in[\mathbf{x}](\cdot), \mathbf{z} \in[\mathbf{z}], \varphi(\mathbf{z}, \mathbf{x}(t)) \in[\varphi]([\mathbf{z}],[\mathbf{x}](t))$ and $\psi(\mathbf{z}, \mathbf{x}(t)) \in[\psi]([\mathbf{z}],[\mathbf{x}](t))$, we get

$$
\forall t \geq 0,0 \neq \varphi(\mathbf{z}, \mathbf{x}(t)) \vee \psi(\mathbf{z}, \mathbf{x}(t))>0 .
$$

Assume now that the left hand side of $(18)$ is also true. From [10 ii), we get that for a specific $t_{0} \in\left[t_{1}, t_{2}\right]$,

$$
\varphi\left(\mathbf{z}, \mathbf{x}\left(t_{0}\right)\right)=0 \wedge \psi\left(\mathbf{z}, \mathbf{x}\left(t_{0}\right)\right) \leq 0
$$

which is not consistent with the previous proposition.

Penumbra test. Given $t_{1}, t_{2} \geq 0$, two trajectories $\mathbf{x}^{a}(\cdot), \mathbf{x}^{b}(\cdot) \in[\mathbf{x}](t)$ and inclusion functions $[\varphi],[\psi]$ for $\varphi, \psi$. Define the penumbra test $T_{\text {penumbra }}([\mathbf{z}])$ as

$$
\left\{\begin{array}{c}
\forall t,[\varphi]\left([\mathbf{z}], \mathbf{x}^{a}(t)\right) \neq 0 \vee[\psi]\left([\mathbf{z}], \mathbf{x}^{a}(t)\right)>0 \\
{[\varphi]\left([\mathbf{z}], \mathbf{x}^{b}\left(t_{1}\right)\right) \cdot[\varphi]\left([\mathbf{z}], \mathbf{x}^{b}\left(t_{2}\right)\right) \leq 0} \\
\wedge \forall t \in\left[t_{1}, t_{2}\right],[\psi]\left([\mathbf{z}], \mathbf{x}^{b}(t)\right) \leq 0
\end{array}\right.
$$

From Theorems 3 and 4 , we get that $T_{\text {penumbra }}$ implies that neither $T_{\text {clarity }}$ nor $T_{\text {dark }}$ will able to conclude anything for any subbox of $[\mathbf{z}]$. 


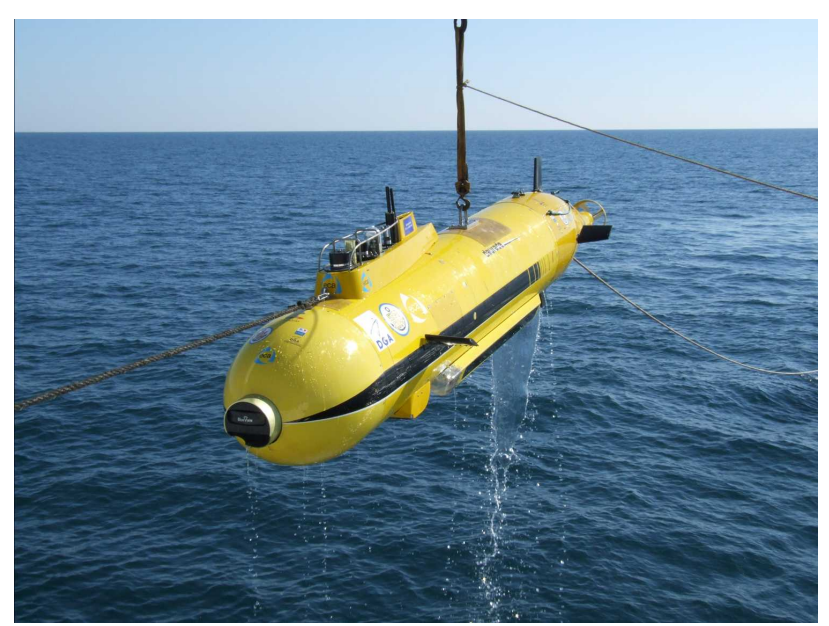

Fig. 5. Daurade: the underwater robot used for the experiment. The portside lateral sonar antenna corresponds to the one meter black segment at the bottom left of the robot

\section{EXPERIMENT}

This section illustrates our sweep exploration method on a real experiment. November 2015, a 46 minutes mission has been performed in the Road-stead of Brest (France, Brittany) with the underwater robot, named Daurade, which realized a classical survey pattern composed of a set of parallel tracks with an altitude of about 10 meters. This robot (see Figure 5) has been built by ECA robotics and used by DGA Tn (Direction Général de l'Armement - Techniques Navales) and SHOM (Service Hydrographique et Océanographique de la Marine) for performing REA (Rapid Environment Assessment) studies. REA is intended to survey the environmental conditions of a particular location in order to identify any existing or potential dangers. In the counter mine warfare context, attention is focused on mapping the sea floor with acoustic sensors. Daurade is equipped with a Side Scan Sonar (Klein 5500) used to detect potential mines. With this sonar, data are recorded on a line perpendicular to the path of the sensor and images are formed by putting side by side these lines. Characterizing the zone seen by the sonar enters inside the framework of sweep exploration.

For the navigation, Daurade relies an inertial central (Phins II IXBlue) coupled with a DVL (Doppler Velocity Log), which returns the speed with respect to the ground. Once under the water, no GPS data are available and the estimated position of the robot drifts with the time. A key point of REA missions is to guarantee that the area of interest has been totally explored without any gap. Assessment of the covered area is usually done manually by an operator who looks at the sonar images. The proposed algorithm can be used to validate the mission plan or, at the end of the mission, to check the area to be explored has indeed been covered.

The horizontal kinematic model the robot is taken as

$$
\left(\begin{array}{c}
\dot{x}_{1} \\
\dot{x}_{2}
\end{array}\right)=\left(\begin{array}{cc}
\cos \psi & -\sin \psi \\
\sin \psi & \cos \psi
\end{array}\right) \cdot \mathbf{v}
$$

where $\left(x_{1}, x_{2}\right)$ corresponds to the $2 \mathrm{D}$ coordinates of the center of the robot expressed in an absolute inertial frame, $\psi$ is the

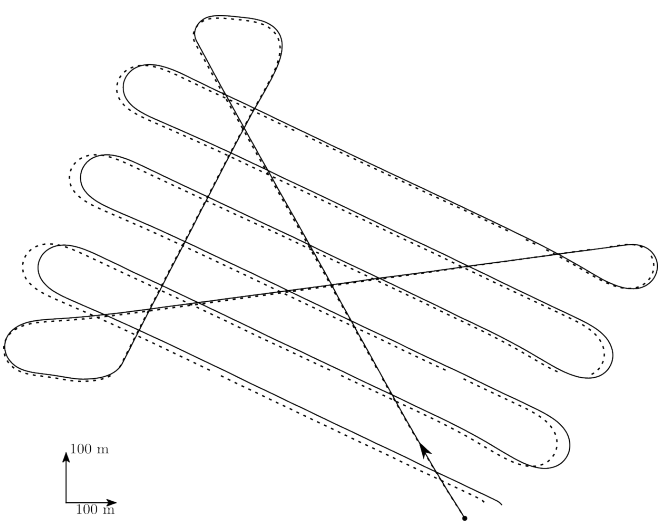

Fig. 6. Online estimated trajectory (plain) and true trajectory (dotted line)

heading and $\mathbf{v}$ is the horizontal speed vector of the robot expressed in its own coordinate system. The aperture angle of the side scan sonar is taken as $\alpha=80^{\circ}$. This means that if the robot has an altitude $a$ then the sonar is able to sense the part of the environment which is perpendicular to the robot and at a distance less than $\ell=a \cdot \sin \alpha$. The horizontal online estimated trajectory is depicted on Figure 6 Note that due to the fact that the controller uses this trajectory for control, it looks perfect, which is not the case for the true trajectory (dotted line) directly measured by an USBL (Ultra-Short Base Line). Now, in our experiment, this true trajectory is neither used for control nor for estimation of the explored zone. It is only used for the validation of the results.

We assume that the initial position is $x_{1}=x_{2}=0$. Taking the interval uncertainties into account and using a guaranteed integration of the state equation 20, we are able to compute a tube which contains the true trajectory. At the end of the mission the position error is around 17 meters. On this experiment, our method provides in less than 5 minutes an estimation of the explored zone as given by Figure 7 . This result is consistent with the true trajectory obtained by the USBL and by an observation of the sonar images.

Consider the same experiment, but, instead of having a sweep exploration, we assume that at each $t$ the visible set $\mathbb{V}(t)$ is a disk of radius $a \cdot \sin \alpha$ (instead of a segment). In this case, the explored zone can still be obtained, but we do not need anymore to use the continuity of the trajectory. The resulting enclosure is given on Figure 8 Note that the uncertainty layer (painted gray) is larger in zones when the location of the robot is less accurate. Note also that Figures 8 and 7 look similar (which is consistent with our intuition) except near the initial position (bottom right) where we can observe the difference between the patch (here a disk) exploration and the sweep (here a segment) exploration.

\section{Conclusion}

This paper has presented a new method to characterize the zone $\mathbb{Z}$ explored by a mobile robot. The method only uses the proprioceptive sensors for localization, which makes the approach reliable. The method is indeed not sensitive $(i)$ to outliers (which mainly occur on exteroceptive sensors), (ii) to data association errors, or (iii) to any information resulting 


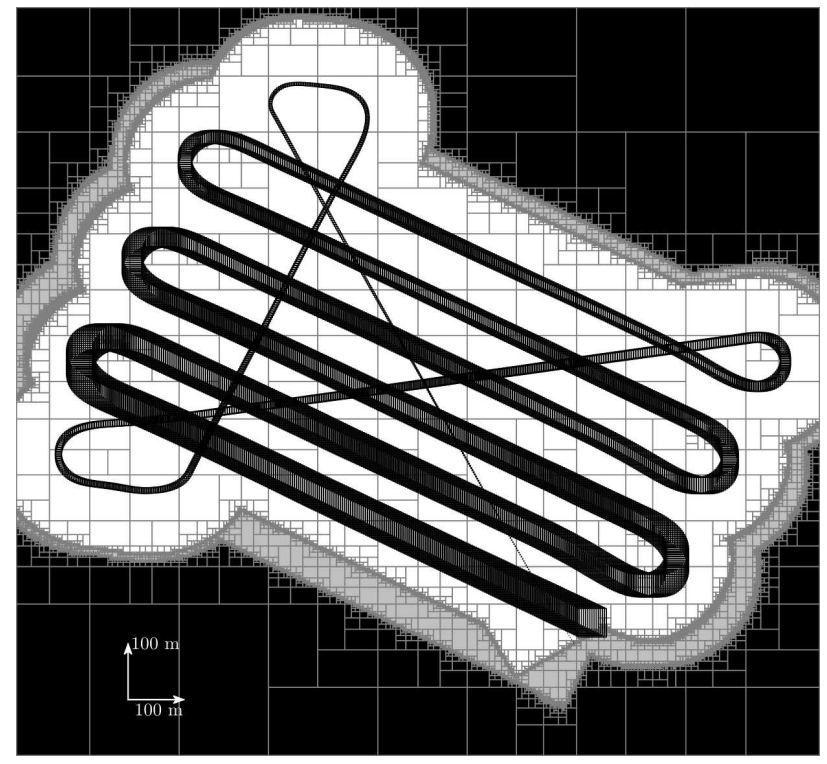

Fig. 7. Enclosure of the explored area. The tube in black encloses the true trajectory

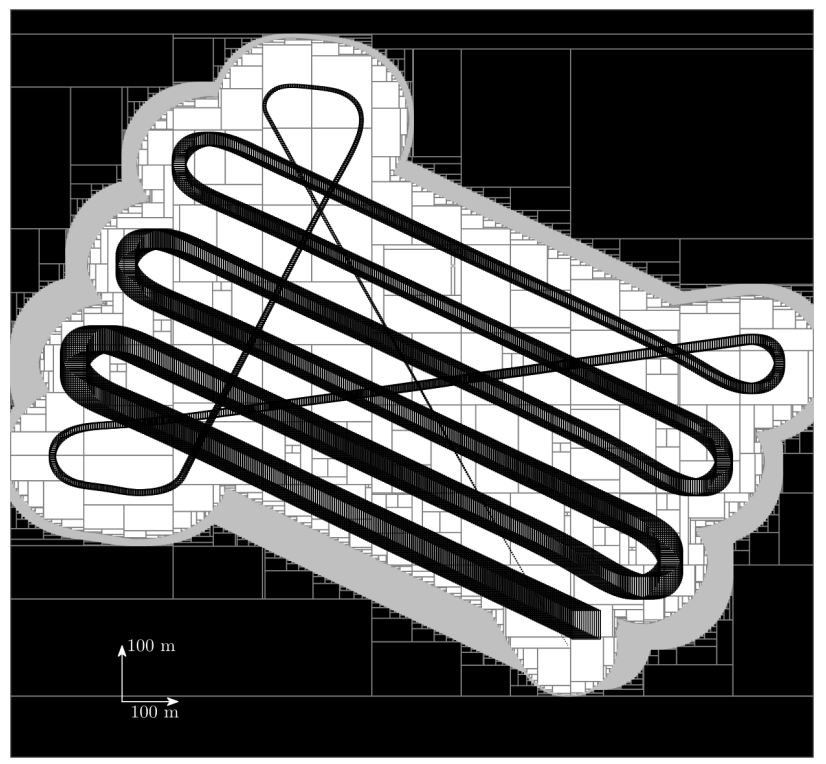

Fig. 8. Patch exploration in the case where the visible sets are disks

from an interaction between the robot and the environment. The method can also be used before the mission in order to guarantee that a given area will all be covered by the robot, whatever the noise on the proprioceptive sensors are. We only need to assume that the state noise is bounded with known bounds in order to be able to enclose the trajectory $\mathbf{x}(\cdot)$ inside a tube $[\mathbf{x}](\cdot)$.

To solve the problem, we had to introduce new interval tests to classify boxes as clear (certainly explored), dark (certainly unexplored) or in the penumbra. This classification requires the development of new quantifier elimination procedures, based on four new theorems. The efficiency of the resulting algorithm has been validated on an actual experiment made by an autonomous underwater robot.

Note. The Python programs, the navigation data made by
Daurade and some videos illustrating the exploration method are given at the following link.

www.ensta-bretagne.fr/jaulin/sweep.html

\section{REFERENCES}

[1] P. Collins and A. Goldsztejn. The Reach-and-Evolve Algorithm for Reachability Analysis of Nonlinear Dynamical Systems. Electronic Notes in Theoretical Computer Science, 223:87-102, 2008.

[2] V. Drevelle, L. Jaulin, and B. Zerr. Guaranteed characterization of the explored space of a mobile robot by using subpavings. In Proc. Symp. Nonlinear Control Systems (NOLCOS'13), Toulouse, 2013.

[3] D. Dubois and H. Prade. Possibility Theory. Plenum, New York, 1988

[4] A. Elfes. Sonar-based real world mapping and navigation. IEEE Transactions on Robotics and Automation, pages 249-265, 1987.

[5] U. Frese. A discussion of simultaneous and mapping. Autonomous Robots, 20:25-42, 2006.

[6] A. Gning and P. Bonnifait. Constraints propagation techniques on intervals for a guaranteed localization using redundant data. Automatica, 42(7):1167-1175, 2006.

[7] R. Guyonneau, S. Lagrange, and L. Hardouin. A visibility information for multi-robot localization. In IEEE/RSJ International Conference on Intelligent Robots and Systems (IROS), 2013.

[8] L. Jaulin. Range-only SLAM with occupancy maps; A set-membership approach. IEEE Transaction on Robotics, 27(5):1004-1010, 2011.

[9] L. Jaulin, M. Kieffer, O. Didrit, and E. Walter. Applied Interval Analysis, with Examples in Parameter and State Estimation, Robust Control and Robotics. Springer-Verlag, London, 2001.

[10] M. Langerwisch and B. Wagner. Building variable resolution occupancy maps assuming unknown but bounded sensor errors. In IEEE/RSJ International Conference on Intelligent Robots and Systems (IROS), Tokyo Big Sight, Japan, 2013.

[11] J. J. Leonard and H. F. Durrant-Whyte. Dynamic map building for an autonomous mobile robot. International Journal of Robotics Research, 11(4), 1992

[12] I. Molchanov. The Theory of Random Sets. Springer, New York, 2005.

[13] John Stephen Mullane, Ba-Ngu Vo, Martin David Adams, and Ba-Tuong Vo. Random Finite Sets for Robot Mapping \& SLAM: New Concepts in Autonomous Robotic Map Representations. Springer Publishing Company, Incorporated, 2013.

[14] O. Mullier, E. Goubault, M. Kieffer, and S. Putot. General inner approximation of vector-valued functions. Reliable Computing, 18(0):117-143, 2013.

[15] L. Paull. Robust Online Adaptive Sensor-Driven Survey Planning for Single and Multiple Autonomous Underwater Vehicles. PhD dissertation, University of New Brunswick, 2013.

[16] N. Revol, K. Makino, and M. Berz. Taylor models and floating-point arithmetic: proof that arithmetic operations are validated in COSY. Journal of Logic and Algebraic Programming, 64:135-154, 2005.

[17] W. Tucker. A Rigorous ODE Solver and Smale's 14th Problem. Foundations of Computational Mathematics, 2(1):53-117, 2002.

[18] D. Wilczak and P. Zgliczynski. Cr-lohner algorithm. Schedae Informaticae, 20:9-46, 2011. 